\title{
Probabilistic Shot Boundary Detection Using Interframe Histogram Differences *
}

\author{
Alvaro Pardo ${ }^{1,2}$ \\ ${ }^{1}$ DIE, Facultad de Ingeniería y Tecnologías, Universidad Católica del Uruguay \\ ${ }^{2}$ IIE, Facultad de Ingeniería, Universidad de la República \\ apardo@ucu.edu.uy
}

\begin{abstract}
In this paper we study a method for the detection of shot boundaries using interframe histogram differences. Instead of using traditional distance between histograms, we use a probabilistic distance that indicates the chance of a given distance to be a shot boundary. We declare a shot change when the interframe histogram difference is a large deviation from the expected histogram interframe differences given past evidence. Like other histogram based methods the proposed one is very simple while being very robust and effective. The proposed method outperforms similar methods proposed in the literature for the detection of hard cuts and achieves good recall and precision performances for gradual transitions.
\end{abstract}

\section{Introduction}

Shot boundary (SB) detection algorithms are one of the most important methods for video analysis. They allow the segmentation of the original video sequence into basic units called shots that facilitate high level processing and abstraction of the video signal. Although it may seem a simple task, the automatic and reliable extraction of $\mathrm{SB}$ it has some difficulties, mainly due to the different types of video sequences. Since a video sequence contains a great amount of data, we should avoid unnecessarily complicated methods. For this reason we will explore possible improvements of classical and simple methods. In this work we will present a simple online method based on color histograms with only a few parameters that performs well for a representative set of tested video sequences.

Existing methods for SB detection can be divided into the following basic categories: pixel, histogram, block matching, object segmentation and tracking, and feature tracking based methods. Pixel based methods usually compute interframe differences between frames (adjacent or not). The main drawback of pixel-based methods is their sensitivity to camera and object motion and noise. For this reason filtering is usually applied before computing interframe differences [1]. Regarding the measure of difference, we can make a distinction between distance based methods and thresholding ones. Usually these methods are not

* Supported by Proyecto PDT-S/C/OP/17/07. 
very reliable and therefore are mostly used as indicators of probable SB that are the confirmed by more sophisticated methods [2].

Histogram based methods compare the histograms of a pair of frames using a suitable histogram distance [3. In contrast to pixel based methods, histogram based methods are robust against camera and object motions since histograms do not contain any spatial information. Unfortunately, the main limitation is that frames of different shots can have similar histograms and in this way these methods will fail. In addition, like pixel-based methods, these methods are not robust against lighting changes. Strong color changes in part of the image may produce false detections.

Block-matching methods divide each frame into blocks and then match a given set of block features (pixel colors, histograms, and so on) between frames. That is, the best match for each block in the source frame is found in the destination frame and the similarity of these block is used as an indicator for SB existence 31 .

Segmentation and object tracking are typically computational demanding. The main idea behind these methods is that frames within a shot contain the same objects, therefore, they use algorithms for object tracking and segmentation to achieve SB detection.

Feature tracking methods detect shot transitions when there is an abrupt change in the number of features tracked. For example, if the frame edges have strong variations [1]. In [4 the authors propose feature tracking as a measure of frame dissimilarity. Instead of tracking edges, they propose to track fine grained features as corners and textures. SB are then detected as points with high interframe feature loss.

Nearly all of the previous methods rely on a set of thresholds in order to decide whether there is a SB in a given frame. The problem of selection of the right threshold is a key point that has big influence in the overall system performance. Unfortunately, it has received little attention in the literature [1 and most of the authors propose heuristics for their selection. Furthermore, it has been demonstrated that global thresholds led to sub optimal methods, with too many false positives or false negatives [1. To solve this problem adaptive thresholds have been proposed. However, when using adaptive thresholds we must design an updating rule based on, for example, the statistics of non-boundary frames. This introduces additional problems concerning the correct estimation of this statistical information. Traditionally the problem is solved introducing a learning stage where several video sequences are processed to obtain the desired statistics.

In this paper we introduce a simple method for the detection of SB using only interframe histogram differences. The method is inspired in the works of Computational Gestalt 56 6 . The key idea in this framework is to define a meaningful event as large deviation from a known background process. Here we will extend those ideas to label a SB when its interframe histogram differences have little probability to be produced given past interframe histogram differences of non-SB frames. The use of past information of non-SB differs from other existing methods which usually evaluate distances between adyacent histograms. This is 
a difference between our proposal and other existing probabilistic histogram (or probability distribution) distance. Also, the use on non-SB allows using a nonsupervised technique; we do not use any training step considering known SB information. Even though the mentioned limitations of histogram based methods will show that they achieve reasonable results. Furthermore, they are less sensitive to compression artifacts as some of the other discussed methods (feature and pixel based for example). In our case all sequences are compressed in mpeg format; some of them with strong compression artifacts.

In the first step of the algorithm we compute a measure of SB probability or meaningfulness. Then in a second stage we apply an adaptive thresholding technique that only uses the information of the video sequence being processed to find the SB. This makes our method very simple and fast.

\section{Proposed Method}

As we said in the previous section our method is based on interframe histogram differences. In what follows we will consider histograms of image pixels computed over a predefined set of bins. Let $H_{i}(t)$ be the histogram of the $i$ color component of the frame 1 , and $H_{d}$ the histogram of (color) histogram differences computed also over a predefined set of bins. That is, if we define $d=\left|H_{i}(t+1)-H_{i}(t)\right|$, $H_{d}$ will contain the histogram of $d$ for previous frames. That is, in each bin it counts the number of points (bins from $H_{i}$ ) where the difference falls within it.

Now let $P_{\mu}=$ Probability $(d(b)>\mu)$ be the probability of the interframe histogram difference at bin $b$ to be above $\mu$. If there is no shot change we expect the histogram differences to be small, and in agreement with previous histogram differences. On the other hand, when a shot change occurs the histogram differences do not follow previous evidence.

Given a fixed threshold $\mu$ we can compute the number of bins in $d$ exceeding the threshold $\mu, N_{\mu}$. To obtain a probabilistic measure of shot change meaningfulness we follow [5] and compute its probability of occurrence given previous interframe histogram differences. This can be done computing the probability of at least $N_{\mu}$ bins out of the total number of bins $N_{b}$ exceeding the threshold $\mu$ by using the Binomial distribution:

$$
B\left(N_{b}, N_{\mu}, P_{\mu}\right)=\sum_{k=N_{\mu}}^{N_{b}} C_{k}^{N_{b}} P_{\mu}^{k}\left(1-P_{\mu}\right)^{N_{b}-k}
$$

Using this probability, we say that the actual histogram difference is meaningful if its probability is very low given previous histogram differences 2. This means that we say that the event is meaningful if it is a large deviation of what is expected given past information.

\footnotetext{
${ }^{1}$ In this work we use the YUV color space.

${ }^{2}$ In the computational gestalt theory instead of working only with the probabilities the authors propose to estimate the expectation via multiplying the probability by the number of test performed 6 .
} 
Finally, the meaningfulness of a histogram difference is obtained as the minimal probability over a set of fixed thresholds:

$$
M=\min _{\mu_{i}} B\left(N_{b}, N_{\mu_{i}}, P_{\mu_{i}}\right)
$$

where each term corresponds to a threshold $\mu_{i} \in\left\{\mu_{1}, \ldots, \mu_{n}\right\}$. The smallest threshold $\mu_{1}$ is estimated from the past values of histogram differences using an $\alpha-\beta$ filter. The upper threshold is set to a reasonable value. In this work we set $\mu_{n}=512$. According to our experiments this parameter does not influence the results.

The probabilities $P_{\mu}$ are obtained from past histogram differences. To deal with non-stationary statistics we use an $\alpha-\beta$ filter. The histogram $H_{d}$ is updated with the following rule:

$$
H_{d}(t+1)=\alpha \operatorname{Histogram}(d(t))+(1-\alpha) H_{d}(t)
$$

with $\alpha=0.2$.

For the computation of the binomial distributions we use the Hoeffding approximations [7] to obtain an upper bound for the logarithm of $M$ when $k / n \geq p$ using ${ }^{3}$ :

$$
\log (B(k, n, p)) \leq k \log \left(\frac{p n}{k}\right)+n\left(1-\frac{k}{n}\right) \log \left(\frac{1-p}{1-\frac{k}{n}}\right)
$$

Since we propose an online method we must decide the occurrence of a shot change using only past values. In fact we introduce a delay in the response in order to consider a window, $W=[t-4, . . t+4]$, centered in $t$. We will say that there is a SB at frame $t$ if the following conditions are fulfilled: $M(t)=\min _{s \in W} M(s)$, $M(t)<\delta \min _{s \in\{t-4, . ., t-1\}} \hat{M}(s)$ or $M(t)<\delta \min _{s \in\{t+1, . ., t+4\}} \hat{M}(s), M(t)<$ $\gamma$ Threshold $(t)$ where $\hat{M}$ are the local maxima of $M$ and Threshold( $t$ ) adaptively computed using only the accumulated values for non-SB $X$ [1]

$$
\operatorname{Threshold}(t)=\alpha \operatorname{Threshold}(t-1)+(1-\alpha) \operatorname{Mean}(X)
$$

For the all the results presented in next section we set: $\delta=4, \gamma=10$ and $\alpha=0.01$.

\section{Results and Evaluation}

To validate the results of the proposed method we are going to test our algorithm against a set of sequences used in [4] where a method for hard cut detection was presented. In figure 1 we show the first frame and the outcome of the proposed method for three sequences. As we can see there are a set of well defined peaks that correspond to SB. In table 1 we present the our results restricted to hard

${ }^{3}$ Since the probabilities are very small is more combinient to work with logarithms. 
Table 1. Comparison for the detection of hard cuts for sequences in figure 1

\begin{tabular}{|c||c|c|c|c|c|c|c|c|c|c|c|c|c|}
\hline \multicolumn{1}{|c|}{} & \multicolumn{1}{|c|}{ Proposed Method } & \multicolumn{2}{|c|}{ Feature tracking } & \multicolumn{3}{|c|}{ Pixel based } & \multicolumn{3}{|c|}{ Histogram based 8} \\
\hline Seq & Prec & Rec & F1 & \multicolumn{1}{|c|}{ Prec } & Rec & F1 & Prec & Rec & F1 & Prec & Rec & F1 \\
\hline \hline $\mathrm{A}$ & 1 & 1 & 1 & 1 & 1 & 1 & 1 & 1 & 1 & 1 & 1 & 1 \\
$\mathrm{~B}$ & 1 & 1 & 1 & 1 & 1 & 1 & .825 & .825 & .825 & 1 & .375 & .545 \\
$\mathrm{C}$ & .946 & .898 & .922 & .595 & .870 & .707 & .764 & .778 & .771 & .936 & .536 & .682 \\
$\mathrm{D}$ & 1 & 1 & 1 & 1 & 1 & 1 & 1 & 1 & 1 & 1 & .941 & .969 \\
$\mathrm{E}$ & 1 & .957 & .978 & .938 & 1 & .968 & .867 & .867 & .867 & .955 & .700 & .808 \\
$\mathrm{~F}$ & 1 & 1 & 1 & 1 & 1 & 1 & 0 & 0 & 0 & 1 & 1 & 1 \\
$\mathrm{G}$ & 1 & 1 & 1 & .810 & .944 & .872 & .708 & .994 & .809 & 1 & .666 & .800 \\
$\mathrm{H}$ & .769 & .976 & .860 & .895 & .895 & .895 & .927 & 1 & .962 & .971 & .895 & .932 \\
$\mathrm{I}$ & 1 & 1 & 1 & 1 & 1 & 1 & 1 & 1 & 1 & 1 & .500 & .667 \\
\hline Average & .968 & .981 & .973 & .915 & .968 & .938 & .788 & .829 & .804 & .985 & .735 & .823 \\
\hline Variance & .006 & .001 & .002 & .019 & .003 & .010 & .099 & .104 & .099 & .001 & .055 & .027 \\
\hline Std dev & .077 & .035 & .050 & .137 & .052 & .100 & .314 & .323 & .315 & .025 & .234 & .165 \\
\hline
\end{tabular}

cuts for comparison purposes with [4. As in [4] we measure the performance of our method using precision (Prec), recall (Rec) and F1 defined as:

$$
\text { Prec }=\frac{\mathrm{T}+}{\mathrm{T}++\mathrm{F}+}, \operatorname{Rec}=\frac{\mathrm{T}+}{\mathrm{T}++\mathrm{F}-}, \mathrm{F} 1=\frac{2 \times \operatorname{Prec} \times \operatorname{Rec}}{\operatorname{Prec}+\operatorname{Rec}}
$$

where $T+, F+$ and $F-$ means true positives, false positives and false negatives respectively.

The precision of the proposed method (PM) outperforms, on average, the feature tracking $(\mathrm{FT})$ and pixel based $(\mathrm{PB})$ methods while performs close to the histogram based (HB) one. On the other hand, considering the recall capabilities, PM outperforms, on average and for each sequence, the precision of the other three methods. Finally, the F1 measure of PM outperforms on average and for each sequence but the $H$, the results of the other methods. Looking at the individual sequences, the only sequence where PM is defeated is sequence $\mathrm{H}$. For this sequence, the precision is worse than other three methods while its recall outperforms FT and HB methods and is close to the PB one.

We conclude that PM outperforms (specially looking at the F1 figures) the other three methods reported for the same sequences. The sequence $\mathrm{H}$ is a problematic case with too many false positives. These false positives are located around frame 3000 where strong lightning change occur, and may be eliminated using a better detection strategy. Particularly, a strategy adapted for this sequence. In our case we used the same strategy (with the same parameters) for all sequences.

When considering the detection of gradual transitions, PM detected all of them in the tested sequences achieving $100 \%$ of precision and recall. Although the obtained results seem very promising the total number of gradual transition considered is not big enough to expect this kind of performance in every case. The numbers of gradual transitions for each sequence are: A 1, C 2, E 4, F 2 and $\mathrm{G}$ 2. However, it is important to note that the detected gradual transitions are of different type. For example, even though the transitions in sequence $\mathrm{F}$ expand along several frames the output of the proposed method has two clear peaks at boundary positions, see figure 1 . 


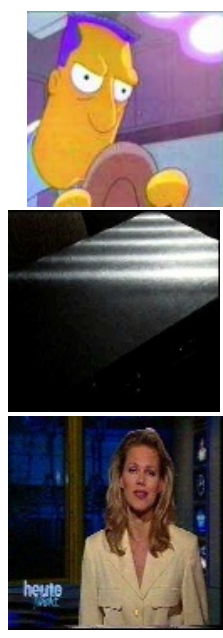

(a)

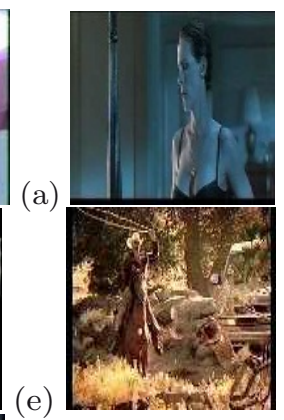

(e)

(b)

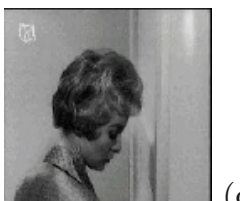

(b)

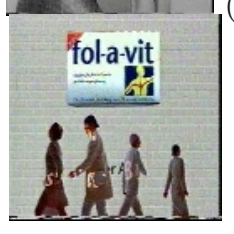

(c)

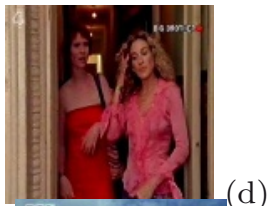

(f)

(g)

(h)

(i)
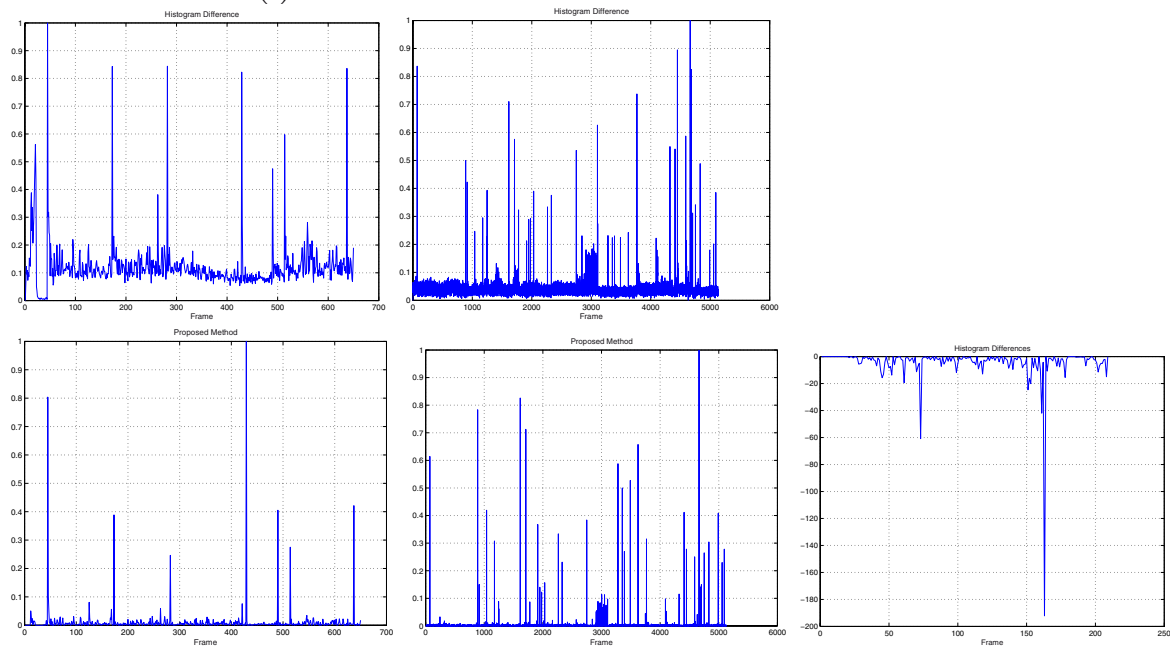

Fig. 1. Top: Sequences. A (Lisa): Cartoon video with substantial object motion. B(Jamie): Strong motions. C(Psycho): Black and white movie with substantial action and motions and many close hard cuts. $\mathrm{D}$ (Sex in the city): High quality digitalization TV show. E(Highlander): Low quality digitalization of TV show. F(TV Commercial): TV Commercial with only a dissolve. G(TV Commercial): TV commercial with hard cuts and gradual transitions. H(Video): Movie with strong lightning changes. I(News): TV news program. Bottom: Comparison of proposed method (meaningfulness $\mathrm{M}$ ) and standard bin-wise histogram difference for sequences A and H. Results for sequence F.

To exemplify the advantages of PM against traditional histogram based methods we compare the outputs of PM and standard histogram difference. We normalized both results dividing each one by its maximum. The results are presented 
in figure 1 for videos A and $\mathrm{H}$. As we can see the results of PM are less noisy and the peaks at SB positions are clearly separated from non-SB ones.

\section{Conclusions and Future Work}

We have presented a simple method that uses histogram differences and improves the results of previously reported methods. The method obtains a measure for shot change meaningfulness with clear peaks at SB. This allows for simpler adaptive threshold and offline detection methods. We formulated the problem inspired in the computational gestalt theory and presented a novel method to compute shot changes based on histogram differences. In future work we will address the limitations of the method with respect to strong color and lightning changes together with better detection strategies.

\section{References}

1. Hanjalic, A.: Shot-boundary detection: Unraveled and resolved. IEEE Transactions on Circuits and Systems for Video Technology (2002)

2. Huang, C.L., Liao, B.Y.: A robust scene-change detection method for video segmentation. IEEE Transactions on Circuits and Systems for Video Technology (2001)

3. Gargi, U., Kasturi, R., Strayer, S.H.: Performance characterization of video-shotchange detection methods. IEEE Transactions on Circuits and Systems for Video Technology (2000)

4. Whitehead, A., Bose, P., Laganiere., R.: Feature based cut detection with automatic threshold selection. In: Proceedings of the International Conference on Image and Video Retrieval. (2004) 410-418

5. Desolneux, A., Moisan, L., J.-M-Morel: A grouping principle and four applications. IEEE Transactions on Pattern Analysis and Machine Intelligence 25 (2003) 508-512

6. Desolneux, A., Moisan, L., J.-M-Morel: Maximal meaningful events and applications to image analysis. The Annals of Statistics 31 (2003) 1822-1851

7. Desolneux, A.: Evnements significatifs et applications l'analyse d'images. $\mathrm{PhD}$ thesis, ENS-Cachan, France (2000)

8. Pfeiffer, S., Leinhart, R., Kuhne, G., Effelserberg, W.: The MoCa Project - Movie Content Analysis REsearch at the University of Mannheim. In: Informatik 1998. (1998) 329-338 